DOI: https://doi.org/10.11144/Javeriana.upsy19.veie

\title{
Validación de las Escalas de Inhibición y Excitación Sexual (SIS/SES-SF) en colombianos *
}

\section{Validity of the Sexual Inhibition/ Sexual Excitation Scales (SIS/SES- $\mathrm{SF}$ ) in Colombians}

\author{
Alejandro SaAvedra-Roa \\ Fundación Universitaria Konrad Lorenz, Colombia \\ ORCID: https://orcid.org/0000-0003-0779-3683 \\ Pablo Vallejo-Medina ${ }^{a}$ \\ Fundación Universitaria Konrad Lorenz, Colombia \\ ORCID: https://orcid.org/0000-0002-8527-1584
} $\begin{array}{lrrr}\text { a Autor } & \text { de correspondencia. Correo } \\ \text { electrónico: } & \text { pablo.vallejom@konradlorenz.edu.co }\end{array}$

Para citar este artículo: Saavedra-Roa, A., \& VallejoMedina, P. (2020). Validación de las Escalas de Inhibición y Excitación Sexual (SIS/SES-SF) en colombianos. Universitas Psychologica, 19, 1-14. https://doi.org/10.11144/Javeriana.upsy19.veie

\section{RESUMEN}

Las escalas SIS/SES-SF han sido desarrolladas para medir la inhibición y la excitación sexual. El objetivo del presente estudio fue adaptar y validar estas escalas en población colombiana. Para esto, se realizó la adaptación cultural y validación de contenido de las escalas por medio del juicio de siete expertos, y se validaron psicométricamente sobre una muestra de 2125 participantes (1000 hombres y 1125 mujeres). Los hombres contestaron al International Index of Erectile Function y la Premature Ejaculation Diagnostic Tool; mientras que el total de la muestra contestó al Sexual Desire Inventory, al Massachusetts General Hospital-Sexual Functioning Questionnaire y a la Sexual Inhibition/Sexual Excitation Scales-Short Form, todo ello junto a una batería sociodemográfica. El muestreo se realizó de forma virtual a través de Facebook. Los índices de las escalas mostraron una apropiada validez y fiabilidad. Además, para ambos sexos, las escalas mostraron un nivel de invarianza estricto. Las relaciones significativas con las otras medidas dan cuenta de su validez de criterio. Se sugiere el uso del instrumento para continuar con la investigación del Modelo de Control Dual dentro de la sexualidad en Latinoamérica.

\section{Palabras clave}

inhibición sexual; excitación sexual; SIS/SES-SF; validez; fiabilidad.

\begin{abstract}
The SIS/SES-SF scales have been developed to measure sexual inhibition and excitation. Current research objective was to adapt and validate these scales in Colombian population. For this purpose, the cultural adaptation and validation of the content of the scales was carried out through the judgment of 7 experts and they were validated on a sample of 2,125 participants (1,000 men and 1,125 women). Men answered the International Index of Erectile Function and the Premature Ejaculation Diagnostic Tool; while the entire sample answered the Sexual Desire Inventory, the Massachusetts General Hospital-Sexual Functioning
\end{abstract}


Questionnaire, and the Sexual Inhibition/Sexual Excitation Scales-Short Form, all of which were accompanied by a socio-demographic battery. The sampling was done virtually through Facebook. The scale indices showed appropriate validity and reliability. In addition, the scales showed a strict level of invariance across sex. Significant relationships with the other measures indicate appropriate criterion validity. The SIS/ SES-SF may be used in order to continue research on the Dual Control Model within sexuality in Latin America.

Keywords

sexual inhibition; sexual excitation; SIS/SES-SF; Colombia; Spanish; validity.

El Modelo de Control Dual (MCD) es un constructo que se fundamenta en la teoría de activación e inhibición conductual de Gray (1987). En este, se propone que la respuesta sexual es el resultado de la interacción homeostática y relativamente independiente de dos sistemas (Bancroft \& Janssen, 2000). Los sistemas involucrados en la respuesta son el de Excitación (ES) e Inhibición (IS) sexual. El MCD es una hipótesis que intenta explicar los mecanismos neurofisiológicos que participan en la respuesta sexual (Bancroft et al., 2009). Tradicionalmente, el ES se ha entendido como la propensión a excitarse en presencia de un estímulo erótico; por su parte, el IS implica perder la excitación sexual cuando se percibe una amenaza (Bancroft \& Janssen, 2000). La importancia de la excitación sexual radica en la influencia que puede tener en el desempeño sexual de los individuos por ejecución o abstención.

En cuanto a la ejecución, por ejemplo, en la revisión realizada por Velten (2017), se sugiere que la propensión alta en IS y moderada o baja en ES podría facilitar algún tipo de disfuncionamiento sexual y, por tanto, afectar otras respuestas implicadas en el comportamiento sexual (e. g., deseo, funcionamiento eréctil o resolución-eyaculación; Levin, 2017) tanto en mujeres (e. g., Sanders et al., 2008; Sarin et al., 2015; Tavares et al., 2018) como en hombres (e. g., Nolet et al., 2017) en muestras clínicas y no clínicas \#considerando particularidades relacionadas con la orientación sexual o el grupo etario\#. Por su parte, la evidencia ha mostrado que una alta propensión al ES y baja al IS podría incrementar la probabilidad de involucramiento en comportamientos sexuales de riesgo que, como resultado, pueden facilitar la adquisición de Infecciones de Transmisión Sexual o embarazos a temprana edad (Rettenberger et al., 2015; van Lankveld et al., 2014). Por último, y a pesar de existir poca evidencia, la investigación ha ido confirmando la hipótesis de afectación de los sistemas de ES e IS dentro de la violencia sexual. Por ejemplo, Kilimnik y Meston (2016) mostraron que la historia de abuso sexual infantil en mujeres puede llegar a impactar en el ES, lo que podría explicar el fenómeno de erotofobia \#y disfuncionamiento sexual\# en edades tardías (Pulverman \& Meston, 2020). Por su parte, Carvalho et al. (2013) mostraron que, en potenciales agresores sexuales, el IS podría verse alterado, relacionándose esto con dificultades eréctiles y orgásmicas en su muestra.

Para poder evaluar la propensión que propone el MCD en humanos, se desarrollaron las escalas de autoinforme Sexual Inhibition/Sexual Excitation Scales (SIS/SES), que han sido validadas en distintos países (e. g., Dèttore et al., 2013; Gomes et al., 2018; Malavige et al., 2013; Turner et al., 2013; Varjonen et al., 2007) y han probado su validez para predecir respuesta de excitación en hombres (Janssen et al., 2002a, 2002b). En mujeres, las propiedades psicométricas de las escalas fueron adecuadas, pero con pesos dispares en algunos ítems (Carpenter et al., 2008). Debido a lo anterior, en un intento por obtener un instrumento con propiedades psicométricas equivalentes para ambos sexos y hacer del instrumento más costo-efectivo diseñado para evaluar el MCD, surgen las Sexual Inhibition/Sexual Excitation Scales-Short Form (SIS/SES-SF; Carpenter et al., 2010). Esta versión ha sido validada recientemente en Alemania (Rettenberger et al., 2019) y en España (Moyano \& Sierra, 2014a) en donde más adelante confirmó su validez prediciendo excitación subjetiva y objetiva (Sierra et al., 2018). Para información detallada de las propiedades psicométricas de la SIS/SES$\mathrm{SF}$, véase el aparte "Instrumentos". 
El MCD parece tener resultados prometedores que permiten la predicción y comprensión de diversos aspectos que atañen en la sexualidad humana (i. e., funcionamiento sexual, conductas sexuales de riesgo y violencia sexual). Adicionalmente, su comprensión en la población colombiana permitiría dos aspectos: 1) el desarrollo de estrategias de prevención primaria y secundaria de problemáticas relacionadas con el funcionamiento sexual o la ejecución de conductas sexuales de riesgo y 2) la intervención de dificultades en funcionamiento sexual o derivadas de la violencia sexual. Sin embargo, para lograr esto, es importante tener medidas que permitan abordar dicha tendencia a la excitación o inhibición sexual de forma válida, fiable y directa \#las cuales, hasta la fecha y hasta donde conocemos, no se encuentran disponibles en Colombia\#. Aunque, hasta el momento existen instrumentos disponibles en Colombia que abordan la excitación sexual femenina (Vallejo-Medina, Saavedra-Roa et al., 2017), su fundamentación teórica no se enmarca en la comprensión del fenómeno desde el MCD, sino en la identificación de dificultades sexuales en general (incluyendo la excitación). Por su parte, los disponibles en el país para evaluar la excitación sexual en hombres, también se centran en la identificación de dificultades a partir de la respuesta de erección (MarchalBertrand et al., 2016) o de la fase de resolucióneyaculación (Vallejo-Medina, Peñuela-Trujillo et al., 2020)

Teniendo la limitación de instrumentos que evalúen la excitación sexual de forma fiable y que permita la comprensión del fenómeno para el desarrollo de estrategias preventivas e interventivas, el objetivo de este estudio, de corte instrumental (Montero \& León, 2007) es adaptar y validar la versión breve de las SIS/ SES-SF en una muestra de hombres y mujeres colombianos. Más concretamente, se pondrá a prueba la validez de contenido, de constructo -incluida la invarianza por sexos-, la validez de criterio y evaluación de su fiabilidad. Para la validez de criterio se plantean las siguientes hipótesis.
H1. Las subescalas del deseo sexual correlacionarán de forma positiva con la SES, mientras que lo harán de forma negativa con las SIS.

H2. Las puntuaciones del PEDT (al estar invertidas) correlacionarán de forma positiva con las SIS, en especial con la inhibición por miedo al rendimiento.

H3. Las puntuaciones del IIFE correlacionarán de forma negativa con las SIS, en especial con la inhibición por miedo al rendimiento.

H4. Las puntuaciones del MGHSFQ correlacionarán de forma negativa con las SIS, en especial con la inhibición por miedo al rendimiento.

\section{Método}

\section{Participantes}

La muestra estuvo conformada por 2125 colombianos $(47.05 \%$ hombres y $52.95 \%$ mujeres) los cuales se encontraban en un rango de edad entre 18 y 73 años $(M=32.94 ; D E$ $=12.12$ ). El $47.2 \%$ de la muestra residía en la ciudad de Bogotá, el $10 \%$ en Medellín, $4.4 \%$ en Cali, $3.2 \%$ en Barranquilla y el porcentaje restante en otras ciudades del país (e. g., Manizales, Cartagena, Bucaramanga, entre otras). Para más información ver Tabla 1. 
Tabla 1

Datos sociodemográficos del segundo grupo

\begin{tabular}{|c|c|c|c|}
\hline Variables & $\begin{array}{c}\text { Hombres } \\
M(D E) \circ n(\%)\end{array}$ & $\frac{\text { Mujeres }}{M(D E) \circ n(\%)}$ & Contraste \\
\hline Edad & $33.46(13.36)$ & $32.46(10.83)$ & $t(1914)=1.86 ; p=0.06$ \\
\hline Años de estudio & $15.33(3.01)$ & $16.3(2.41)$ & $\begin{array}{c}f(2039)=8.01 ; p<0.01 ; \\
d=0.35\end{array}$ \\
\hline \multicolumn{4}{|l|}{ Orientación sexual } \\
\hline Asexual & $17(1.7)$ & $6(0.6)$ & \multirow{8}{*}{$\begin{array}{c}\chi^{2}(7)=110.05 ; p< \\
0.01 ; n^{2}=0.05\end{array}$} \\
\hline Exclusivamente heterosexual & $724(72.4)$ & $883(82.8)$ & \\
\hline 2 & $62(6.2)$ & $105(9.8)$ & \\
\hline 3 & $11(1.1)$ & $18(1.7)$ & \\
\hline 4 & $16(1.6)$ & $12(1.1)$ & \\
\hline 5 & $22(2.2)$ & $9(0.8)$ & \\
\hline 6 & $33(3.3)$ & $11(1)$ & \\
\hline Exclusivamente homosexual & $115(11.5)$ & $23(2.2)$ & \\
\hline \multicolumn{4}{|l|}{ Al menos 6 meses en una relación } \\
\hline $\mathrm{Si}$ & $606(61.1)$ & $752(70.2)$ & \multirow{2}{*}{$\begin{array}{c}\chi^{2}(1)=19.06 ; p< \\
0.01 ; n^{2}=0.009\end{array}$} \\
\hline No & $386(38.9)$ & $319(29.8)$ & \\
\hline \multicolumn{4}{|l|}{ Estado civil } \\
\hline Casado & $178(17.9)$ & $215(20)$ & \multirow{5}{*}{$\chi^{2}(4)=6.54 ; p=0.16$} \\
\hline Soltero & $598(60.1)$ & $613(57.1)$ & \\
\hline Viudo & $3(0.3)$ & $5(5)$ & \\
\hline Unión de he & $146(14.7)$ & $141(13.1)$ & \\
\hline Separado & $70(7)$ & $100(9.3)$ & \\
\hline Salario mínimo colombiamo & $3.45(2.81)$ & $3.40(2.46)$ & \\
\hline \multicolumn{4}{|l|}{ Asistencia a actos religiosos } \\
\hline Nunca & $315(31.6)$ & $209(19.5)$ & \multirow{6}{*}{$\begin{array}{c}\chi^{2}(5)=42.8 ; p<0.01 ; \\
n^{2}=0.01\end{array}$} \\
\hline Alguna vez al año & $299(30)$ & $398(37.1)$ & \\
\hline Alguna vez al mes & $202(20.3)$ & $260(24.2)$ & \\
\hline Una vez por semana & $133(13.3)$ & $157(14.6)$ & \\
\hline Varias veces por semana & $38(3.8)$ & $43(4)$ & \\
\hline A diario & $10(1)$ & $7(0.7)$ & \\
\hline
\end{tabular}

Nota. $M=$ media; $D E=$ desviación estándar.

\section{Instrumentos}

Información sociodemográfica. Mediante una entrevista semiestructurada se preguntó por una serie de características sociodemográficas de los participantes: edad, sexo, años de estudios, lugar de residencia, orientación sexual (cf., Kinsey et al., 1948), creencias religiosas, asistencia a actos religiosos, estado civil, ingresos salariales, y se indagó sobre si tenían o no una relación afectiva de al menos seis meses de duración.

Sexual Inhibition/Sexual Excitation Scales-Short Form ([SIS/SES-SF]; Carpenter et al., 2010). Para la presente investigación, se hizo uso de la versión española (Moyano \& Sierra, 2014a). Las SIS/SES-SF están conformadas por un total conglomerado de 14 ítems que se responden en una escala tipo Likert de cuatro puntos, así: 1 = Completamente en desacuerdo y 4 = Completamente de acuerdo (Carpenter et al., 2010), los cuales se distribuyen en los siguientes tres factores/escalas: la Escala de Excitación Sexual (SES, por sus siglas en inglés) en relación con las interacciones con otras personas (e. g., "Cuando hablo por teléfono con alguien que tiene una voz sexy, me excito sexualmente."); la Escala de Inhibición por Miedo a Fallar en el Desempeño (SIS-1, por sus siglas en inglés) relacionada con la concentración/distracción durante la actividad sexual y sus consecuencias (e. g., "Cuando algún pensamiento me distrae, pierdo fácilmente mi erección/excitación.") y la Escala de Inhibición por Miedo a las Consecuencias del Desempeño (SIS-2, por sus siglas en inglés) con ítems que tratan la posibilidad de ser descubierto o contraer alguna infección de transmisión sexual (e. g., "Si me doy cuenta de que hay riesgo de contagio de una infección de transmisión sexual, es poco probable que mantenga la excitación sexual."). Los ítems 1, 3, 8, 10, 11 y 14 corresponden al factor/escala SES; los ítems 4, 9 y 12 al SIS- 1 y 2 , 5,6 y 7 al SIS-2. Las puntuaciones oscilan de 6 a 24 puntos para la SES y de 4 a 16 puntos para la SIS-1 y SIS-2.

Sexual Desire Inventory ([SDI]; Spector et al., 1996). Se hizo uso de la versión española (Moyano, Vallejo-Medina \& Sierra, 2016), también validada en Colombia (Vallejo-Medina, Rojas-Paoli et al., 2020). Se trata de una escala de 13 ítems que evalúa el interés por la actividad sexual diádica (i. e., en pareja y/u otra extraña/ sexualmente atractiva) y solitaria. Los alfa de Cronbach de esta versión para mujeres $0.88,0.89$ y 0.93 y para hombres $0.8,0.86$ y 0.9 muestran una alta fiabilidad del instrumento en sus tres dimensiones en el presente estudio (i. e., deseo sexual a la pareja, a una persona atractiva y en solitario). La escala de respuesta variable de tipo Likert, sin embargo, varía de acuerdo al ítem. Un ejemplo de ítem sería: "Cuando ve por primera vez una persona atractiva, iqué tan fuerte es su deseo sexual?".

Massachusetts General Hospital-Sexual Functioning Questionnaire ([MGH-SFQ]; Fava et al., 1998). Para el presente estudio, se utilizó la versión validada en Colombia (MarchalBertrand et al., 2016). Es un autoinforme que consta de cinco ítems, siendo el ítem 4 exclusivamente para hombres. Cada uno de los ítems representa una dimensión de sexualidad: deseo, excitación, orgasmo, erección y satisfacción general. Su escala de respuesta es de tipo Likert de cinco puntos $(1=$ Totalmente 
disminuido/a y $5=$ Normal). Los participantes respondían ítems como: "¿Cómo ha estado su interés sexual durante el último mes?". Los alfas de Cronbach en este estudio fueron de 0.89 y 0.87 para mujeres y hombres, respectivamente.

International Index of Erectile Function ([IIEF-5]; Rosen et al., 1999). La versión en español usada fue la realizada por Pfizer $^{1}$ y validada para Colombia (Vallejo-Medina et al., 2020). Es un cuestionario corto que evalúa la función eréctil en una escala tipo Likert de cinco opciones de respuesta, las cuales cambian de acuerdo al ítem. El puntaje máximo es de 25 puntos, siendo 21 el punto de corte (i. e., puntuaciones superiores indican la ausencia de disfunción eréctil). El alfa de Cronbach para esta investigación fue de 0.81. Un ejemplo de ítem sería: "¿Cómo calificaría su confianza para lograr y mantener una erección?".

Premature Ejaculation Diagnostic Tool ([PEDT]; Symonds et al., 2007). Para el presente estudio, se utilizó la versión en español realizada por Pfizer $^{2}$ también validada en Colombia (Vallejo-Medina, Saffon et al., 2020). Este instrumento evalúa la eyaculación precoz con una escala tipo Likert de cinco opciones de respuesta, las cuales cambian de acuerdo al ítem. La puntuación máxima posible es de 20 , siendo 8 su punto de corte (i. e., puntuaciones inferiores indican ausencia de eyaculación prematura). El alfa de Cronbach en este estudio fue de 0.78. Un ejemplo de ítem sería: "¿Eyacula antes de lo que quisiera?".

\section{Procedimiento}

Un equipo de cuatro psicólogas colombianas que habían residido al menos un año en España se encargó de realizar la adaptación cultural del cuestionario del español que se usa en España al de Colombia bajo las recomendaciones brindadas por Vallejo-Medina, Gómez-Lugo et al. (2017). La adaptación cultural del SIS/SESSF se realizó bajo los lineamientos propuestos por Elosua et al. (2014), Muñiz et al., (2013), así como la American Educational Research Association, the American Psychological Association $y$ the National
Council on Measurement in Education (2014).

Las propiedades de los ítems de la versión adaptada del SIS/SES-SF fueron evaluadas por siete psicólogos colombianos que trabajaban en sexualidad humana. Los expertos evaluaron cinco propiedades de los ítems: Representatividad al constructo (i. e., IS o ES), Pertenencia dentro de cada factor (i. e., SES, SIS-1 y/o SIS-2), Comprensión en su versión adaptada, Interpretación respecto al grado de ambigüedad y Claridad (i. e., qué tan conciso es). Los expertos puntuaron lo ítems de 1 (Nada...) a 4 (Muy...) en cada propiedad. Adicionalmente, brindaron una redacción alternativa del ítem si así lo consideraban.

El muestreo fue no probabilístico de tipo incidental y se realizó entre el 16 de septiembre y el 30 de octubre de 2015. Las escalas fueron administradas mediante la plataforma TypeForm (C), la cual permite responder desde cualquier dispositivo electrónico con acceso a Internet. El tiempo promedio de respuesta fue de 24'03". Se utilizó la página institucional de Facebook (C) del laboratorio (Oculto para la revisión) para la distribución de la encuesta. Similar a otros estudios (Vallejo-Medina, Saavedra-Roa et al., 2017), la duplicación de participantes se controló por medio del IP, sexo y edad.

\section{Análisis de datos}

Para el análisis de contenido se utilizó una tabla de especificaciones (Osterlind, 1989) y el programa ICaiken (Merino \& Livia, 2009) el cual permitió calcular el coeficiente de acuerdo entre expertos $V$ de Aiken (Aiken, 1985). Todo ítem que obtuviera puntuación inferior a 0.5 en el límite inferior ( $\mathrm{IC}=95 \%)$ del intervalo $V$ de Aiken sería excluido (Merino \& Livia, 2009). Los comentarios relevantes realizados por los expertos fueron tomados en cuenta para realizar los ajustes pertinentes al ítem.

El alfa ordinal de las SIS/SES-SF, el coeficiente ítem total corregido y el alfa de Cronbach de la escala si se eliminara cada uno de los 
ítems, fueron estimados con el software Factor 10.3.01 01 (Lorenzo-Seva \& Ferrando, 2015). Estos indicadores son adecuados para ítems con respuesta politómica como los del SIS-SES. El software EQS 6.1 se utilizó para confirmar la estructura factorial y la Invarianza Factorial (IF) que se llevó a cabo por medio del procedimiento progresivo de las estructuras de medias y covarianzas como se recomienda ([MACS]; Byrne et al., 2009). Se hizo uso del test de Mardia para obtener la distribución multivariada de los datos (valores superiores a cinco indican no normalidad), dado el incumplimiento de esta, se emplearán métodos robustos.

La IF fue evaluada progresivamente. Se hizo uso del método Maximum Likelihood Robust (ML, Robust) para este procedimiento. Esta se evaluó de la siguiente manera: 1) invarianza configural: sin restricciones en el modelo; 2) invarianza métrica o débil: donde los pesos de cada factor eran restringidos, de esta manera se evaluó la equivalencia de los pesos de cada ítem por factor; invarianza fuerte: los pesos de cada ítem son restringidos y 3) invarianza estricta: las varianzas de los errores son restringidas. Para observar los ajustes del modelo, se tuvo en cuenta el Root Mean Square Error Approximation (RMSEA), el Comparative Fit Index (CFI) y el intervalo de confianza (IC $=90 \%$ ). Puntuaciones inferiores a 0.08 para el RMSEA y superiores a 0.95 para el CFI, fueron indicadores de un ajuste apropiado del modelo. Para dar cuenta de la invarianza, se tomó un decremento no mayor al 0.01 en el CFI con respecto al modelo restrictivo (Cheung \& Rensvold, 2002). Para estimar la bondad del modelo tridimensional de cada una de las invarianzas estimadas, se utilizó el Akaike Information Criterion (AIC).

\section{Consideraciones éticas}

La presente investigación fue revisada y aprobada por el comité de ética independiente de la Facultad de Psicología de la Fundación Universitaria Konrad Lorenz dentro del documento "Informes de Investigaciones 2014 Acta número 2015-009”, siguiendo la
Declaración de Helsinki de 1975, revisada en 1983 por el Comité de Ética de Investigación Clínica. Todos los participantes aceptaron voluntariamente participar en la investigación. El comité de ética revisó el proceso de consentimiento, pero no el documento en sí mismo.

\section{Resultados}

La Tabla 2 muestra la evaluación cualitativa realizada por los siete expertos en sexualidad humana en los 14 ítems adaptados para Colombia. Todas las puntuaciones en la $V$ de Aiken fueron superiores a 0.5 en el límite inferior del IC. De esta manera, los ítems de las SIS/SESSF mostraron adecuada redacción y adaptación.

Tabla 2

Evaluación de características de los ítems de la SIS/ SES-SF

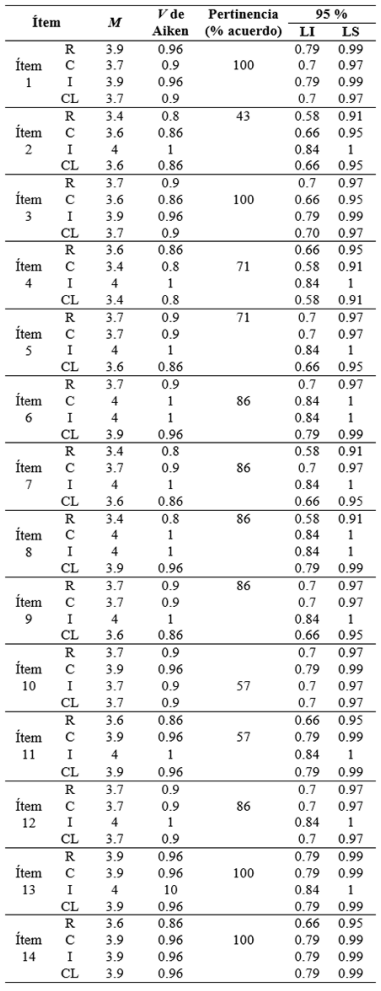

Note: $\mathrm{R}=$ Representatividad; $\mathrm{C}=$ Comprensión; $\mathrm{I}=$ Interpretación; $\mathrm{CL}=$ Claridad; $\mathrm{M}=$ Media; LI= Límite inferior; LS= Límite superior. 
Fiabilidad y propiedades psicométricas de los ítems

La Tabla 3 muestra las propiedades de los ítems de las escalas para hombres y mujeres. Las SIS/ SES-SF son fiables con valores similares en ambos sexos. Las correlaciones ítem-total corregidas (ctic) son siempre superiores a 0.3 , excepto para el ítem 6 en ambos grupos. El único incremento considerable $(>0.1)$ en el alfa de Cronbach se da si el ítem cuatro se elimina de la escala SIS-1 en las mujeres. Sin embargo, esta escala ha mostrado ser robusta en otros indicadores, por lo que se decide mantener el ítem. Adicionalmente, se observa que las desviaciones estándar estuvieron cercanas a 1, lo cual supone una adecuada variabilidad de respuesta.

\section{Tabla 3}

Propiedades psicométricas de los ítems para hombres y mujeres colombianos

\begin{tabular}{|c|c|c|c|c|c|c|c|c|c|c|c|}
\hline \multirow[b]{2}{*}{ Escala } & \multirow[b]{2}{*}{ 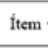 } & \multicolumn{5}{|c|}{ Hombres } & \multicolumn{5}{|c|}{ Mujeres } \\
\hline & & $M(D E)$ & etic & $\alpha-i$ & $M(D E)$ & $\alpha$ & $M(D E)$ & etic & $a-\hat{i}$ & $M(D E)$ & $\alpha$ \\
\hline \multirow{7}{*}{ SES } & 1 & $20.33(0.86)$ & 0.52 & 0.75 & \multirow{6}{*}{$160.48(20.93)$} & \multirow{6}{*}{0.8} & $10.75(0.76)$ & 0.49 & 0.78 & \multirow{6}{*}{$140.08(30.08)$} & \multirow{6}{*}{0.81} \\
\hline & 3 & $20.32(0.83)$ & 0.43 & 0.78 & & & $10.99(0.83)$ & 0.52 & 0.77 & & \\
\hline & 8 & $20.91(0.71)$ & 0.5 & 0.77 & & & $20.39(0.82)$ & 0.55 & 0.77 & & \\
\hline & 10 & $30.09(0.62)$ & 0.4 & 0.79 & & & $20.95(0.68)$ & 0.41 & 0.81 & & \\
\hline & 11 & $30.12(0.68)$ & 0.44 & 0.78 & & & $20.87(0.77)$ & 0.38 & 0.81 & & \\
\hline & 14 & $20.71(0.71)$ & 0.53 & 0.75 & & & $20.13(0.73)$ & 0.54 & 0.76 & & \\
\hline & 4 & $20.16(0.8)$ & 0.41 & 0.77 & & & $20.34(0.85)$ & 0.39 & 0.92 & \multirow{4}{*}{$90.38(20.21)$} & \\
\hline \multirow{3}{*}{ SIS-1 } & 9 & $20.26(0.79)$ & 0.37 & 0.77 & \multirow{3}{*}{$80.77(20.16)$} & \multirow{3}{*}{0.76} & $20.42(0.84)$ & 0.29 & 0.78 & & \multirow{3}{*}{0.77} \\
\hline & 12 & $20.29(0.72)$ & 0.51 & 0.61 & & & $20.41(0.73)$ & 0.51 & 0.63 & & \\
\hline & 13 & $20.05(0.74)$ & 0.5 & 0.62 & & & $20.21(0.76)$ & 0.47 & 0.58 & & \\
\hline \multirow{4}{*}{ SIS.2 } & 2 & $20.34(0.88)$ & 0.4 & 0.63 & & & $20.64(0.95)$ & 0.41 & 0.6 & \multirow{4}{*}{$110.6(20.44)$} & \multirow{4}{*}{0.7} \\
\hline & 5 & $20.53(0.86)$ & 0.32 & 0.7 & \multirow{3}{*}{$100.57(20.32)$} & \multirow{3}{*}{0.67} & $20.74(0.87)$ & 0.36 & 0.9 & & \\
\hline & 6 & $30.19(0.87)$ & 0.21 & 0.65 & & & $30.39(0.81)$ & 0.28 & 0.67 & & \\
\hline & 7 & $20.52(0.92)$ & 0.42 & 0.54 & & & $20.83(0.95)$ & 0.47 & 0.59 & & \\
\hline
\end{tabular}

Nota: $\mathrm{M}=$ media; $\mathrm{DE}=$ desviación estándar; ctic $=$ correlación ítem-total corregida; $\alpha-i=$ alfa de Cronbach si el ítem es eliminada; $\alpha=$ alfa de Cronbach

\section{Invarianza factorial}

Se llevó a cabo una invarianza factorial para evaluar la equivalencia de factores entre sexos (Tabla 4). El test de Mardia mostró una distribución multivariada de 46.65 y 47.96 para mujeres y hombres, respectivamente. Los valores estimados para CFI y el RMSEA en todos los niveles (i. e., configural, débil, fuerte y estricta) cumplieron con los indicadores para estimar el ajuste del modelo (Cheung \& Rensvold, 2002). Adicionalmente, los decrementos de las puntuaciones del CFI no fueron superiores a 0.01 en el cambio de un nivel con respecto al siguiente más restrictivo. En todos los niveles, los valores del RMSEA se mantuvieron menores a 0.08 dentro del intervalo de confianza al $90 \%$. Así, los valores observados tanto en el RMSEA como el CFI para cada nivel de invarianza sugieren un ajuste apropiado de la matriz de datos al modelo teórico propuesto y una equivalencia del mismo y sus errores en ambos sexos. La Figura 1 diagrama los resultados estandarizados del modelo configural, los pesos factoriales asociados $(\lambda)$, los errores de cada ítem y la varianza del ítem explicada por cada factor (i. e., SES, SIS-1 y SIS-2).

\section{Figura 1}

Los valores subrayados corresponden al grupo de hombres y los restantes a las mujeres. Invarianza configural del modelo de la Escala de Inhibición Sexual/Escala de Excitación Versión Corta (SIS/SES-SF) en población colombiana. SES = Escala de Excitación Sexual. SIS-1 = Escala de Inhibición por Miedo a Fallar en el Desempeño. SIS-2 = Escala de Inhibición por Miedo a las Consecuencias. Con el fin de obtener mayor claridad visual se ha suprimido los interceptos (V999).

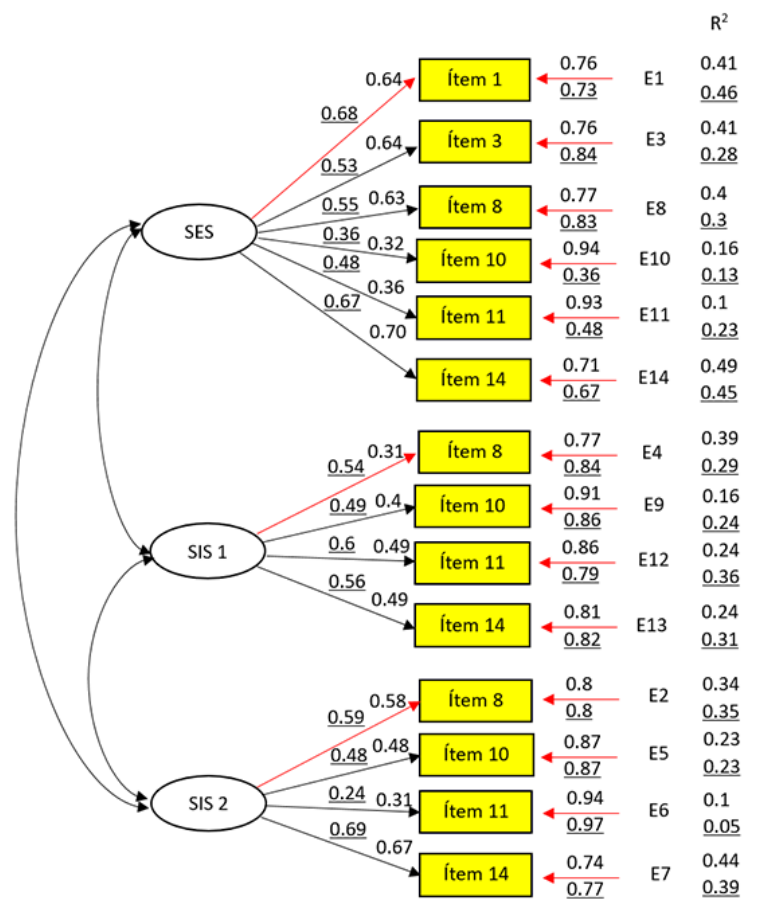


Tabla 4

Índices de Calidad de Ajuste para los Diferentes Modelos de Invarianza

\begin{tabular}{|c|c|c|c|c|c|c|c|c|}
\hline \multirow{2}{*}{$\begin{array}{c}\text { Nivel de } \\
\text { invarianza }\end{array}$} & \multirow{2}{*}{ S-B $\chi^{2}$} & \multirow{2}{*}{$d f$} & \multirow{2}{*}{ AIC } & \multirow{2}{*}{ CFI } & \multirow{2}{*}{$\Delta$ CFI } & \multirow{2}{*}{ RMSEA - } & \multicolumn{2}{|c|}{$90 \%$ for RMSEA } \\
\hline & & & & & & & LI & LS \\
\hline $\begin{array}{l}\text { Invarianza } \\
\text { configural }\end{array}$ & $266.8^{*}$ & 136 & -50.19 & 0.964 & - & 0.031 & 0.026 & 0.037 \\
\hline Invarianza débil & $313.48^{*}$ & 150 & 130.48 & 0.954 & -0.01 & 0.033 & 0.028 & 0.039 \\
\hline Invarianza fuerte & $711.9^{*}$ & 164 & 3830.9 & 0.955 & 0.001 & 0.059 & 0.054 & 0.063 \\
\hline Invarianza estricta & $785.12^{*}$ & 178 & 4290.12 & 0.945 & -0.01 & 0.059 & 0.055 & 0.063 \\
\hline
\end{tabular}

Nota. S-B $\chi^{2}=$ Chí-Cuadraro Escalado Santorra-Bentler; $\mathrm{df}=$ grados de libertad; AIC: Criterio de Información Akaike; CFI = Comparative Fit Index; $\Delta \mathrm{CFI}=$ Incremento del Comparative Fit Index; RMSEA = Root Mean Square Error of Approximation; LI $=$ Límite Inferior; $\mathrm{LS}=$ Límite Superior. $* p<0.001$

Validez de criterio y clasificación de puntuaciones por percentiles

La validez de criterio de las SIS/SES-SF se determinó mediante las correlaciones de Pearson observadas entre sus factores y el PEDT, IIEF-5, MGH-SFQ y el IDS. En la Tabla 5, se muestran las correlaciones positivas y estables entre el factor SES, el funcionamiento sexual general (MGH-SFQ) y el IDS. Correlaciones negativas y significativas se observaron entre los factores inhibitorios (i. e., SIS-1 y SIS-2) y el IDS, MGHSFQ y el IIFE-5, como se esperaba.

\section{Tabla 5}

Correlaciones entre factores del SIS/SES-SF y las otras medidas por sexo

\begin{tabular}{|c|c|c|c|c|c|c|c|c|c|}
\hline Mujeres & PDET & IIEF -5 & MGHSFQ & $\begin{array}{c}\text { IDS } \\
\text { Pareja }\end{array}$ & $\begin{array}{c}\text { IDS } \\
\text { Atractiva }\end{array}$ & $\begin{array}{c}\text { IDS } \\
\text { Solidario }\end{array}$ & SES & sIS1 & sIS2 \\
\hline PDET & & $.0 .35^{* \prime}$ & $-0.23^{*}$ & $-0.07^{*}$ & 0.02 & 0 & $0.06^{\circ}$ & $0.15^{*}$ & $0.12^{*}$ \\
\hline IEEF & . & & $0.42^{* *}$ & $0.22^{*}$ & -0.02 & .0 .06 & -0.04 & $-0.4^{* *}$ & $-0.1^{*}$ \\
\hline MGHSFQ & . & . & & $0.29^{*}$ & 0 & -0.01 & -0.02 & $-0.26^{*}$ & $-0.07^{*}$ \\
\hline IDS Pareja & . & . & $0.56^{* *}$ & & $0.1^{*}$ & $0.12 *$ & $0.1^{* *}$ & $-0.19^{*}$ & $-0.08^{* *}$ \\
\hline IDS Atractiva & . & . & 0.03 & $0.13^{*}$ & & $0.44^{*}$ & $0.54^{* *}$ & $0.09^{* *}$ & -0.04 \\
\hline IDS Solidario & . & . & $0.19^{* \prime}$ & $0.28^{*}$ & $0.41^{*}$ & & $0.42^{*}$ & $0.08^{*}$ & $-0.08^{*}$ \\
\hline SES & . & . & $0.1^{*}$ & $0.19^{*}$ & $0.57^{* *}$ & $0.4^{*}$ & & $0.15^{*}$ & -0.01 \\
\hline sIs1 & . & . & $-0.3^{* *}$ & $-0.23^{*}$ & $.0 .08^{* *}$ & $-0.13^{*}$ & -0.03 & & $0.33^{*}$ \\
\hline sIs2 & . & . & $-0.11 *$ & $-0.16^{* \prime}$ & $-0.2^{* *}$ & $-0.16^{* *}$ & $-0.15^{*}$ & $0.31^{* *}$ & \\
\hline
\end{tabular}

Finalmente, se obtuvieron los rangos de puntuaciones de acuerdo a los percentiles para Colombia, estos fueron diferenciados por sexo y rangos de edad (i. e., 18-30, 31-44 y mayores de 45 años) en cada factor (Tabla 6).

\section{Tabla 6}

Escala de Inhibición Sexual/Excitación Sexual versión corta (SIS/SES-SF) validada para Colombia dividida por sexo, rangos de edad y factores

\begin{tabular}{|c|c|c|c|c|c|c|c|c|c|}
\hline \multicolumn{10}{|c|}{ Mujeres } \\
\hline & \multicolumn{3}{|c|}{ SES } & \multicolumn{3}{|c|}{ SIS-1 } & \multicolumn{3}{|c|}{ SIS-2 } \\
\hline Edades & $18-30$ & $31-44$ & +45 & $18-30$ & $31-44$ & ${ }^{+} 45$ & $18-30$ & $31-44$ & ${ }^{+} 45$ \\
\hline $\mathrm{N}$ & 546 & 246 & 139 & 559 & 253 & 140 & 549 & 254 & 135 \\
\hline$M$ & 14.02 & 14.48 & 13.66 & 9.27 & 9.32 & 9.79 & 11.42 & 11.69 & 12.28 \\
\hline$D E$ & 3.02 & 3 & 3.11 & 2.12 & 2.26 & 2.46 & 2.37 & 2.28 & 2.61 \\
\hline Asimetria & 0.09 & -0.22 & -0.16 & 0.18 & 0.44 & -0.08 & -0.13 & -0.28 & -0.72 \\
\hline Curtosis & 0.08 & 0.09 & -0.04 & 0.21 & 0.29 & 0.16 & -0.32 & 0.11 & 0.67 \\
\hline Minimo & 6 & 6 & 6 & 4 & 4 & 4 & 4 & 5 & 4 \\
\hline Máximo & 24 & 23 & 21 & 16 & 16 & 16 & 16 & 16 & 16 \\
\hline \multicolumn{10}{|l|}{ Percentil } \\
\hline & 9 & 9 & 8 & 6 & 6 & 5 & 7 & 7 & 7 \\
\hline pl5 & 11 & 11 & 10 & 7 & 7 & 7 & 9 & 10 & 10 \\
\hline p25 & 12 & 13 & 12 & 8 & 8 & 8 & 10 & 10 & 11 \\
\hline $\mathrm{p} 3$ & 13 & 13 & 13 & 8 & 8 & 9 & 10 & 11 & 12 \\
\hline p50 & 14 & 15 & 14 & 9 & 9 & 10 & 12 & 12 & 12 \\
\hline p65 & 15 & 16 & 15 & 10 & 10 & 11 & 12 & 12 & 13 \\
\hline p75 & 16 & 16 & 16 & 11 & 11 & 11 & 13 & 13 & 14 \\
\hline p85 & 17 & 18 & 17 & 12 & 12 & 12 & 14 & 14 & 15 \\
\hline p95 & 19 & 19 & 18 & 13 & 13 & 14 & 15 & 16 & 16 \\
\hline \multicolumn{10}{|c|}{ Hombres } \\
\hline & \multicolumn{3}{|c|}{ SES } & \multicolumn{3}{|c|}{ SIS-1 } & \multicolumn{3}{|c|}{ SIS-2 } \\
\hline Edades & $18-30$ & $31-44$ & +45 & $18-30$ & $31-44$ & +45 & $18-30$ & $31-44$ & ${ }^{+} 45$ \\
\hline $\mathrm{N}$ & 504 & 214 & 228 & 506 & 216 & 228 & 507 & 217 & 224 \\
\hline$M$ & 16.38 & 16.79 & 16.34 & 8.53 & 8.86 & 9.28 & 10.32 & 10.78 & 11.01 \\
\hline$D E$ & 3 & 2.98 & 2.71 & 2.23 & 2.15 & 1.99 & 2.38 & 2.17 & 2.26 \\
\hline Asimetria & -0.03 & 0.23 & -0.01 & 0.24 & 0.11 & 0.03 & 0.04 & -0.04 & -0.17 \\
\hline Curtosis & -0.06 & 0.18 & 0.21 & 0.37 & 0.15 & 0.79 & -0.04 & -0.01 & 0.08 \\
\hline Minim & 7 & 7 & 9 & 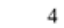 & 4 & 4 & 4 & 5 & 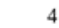 \\
\hline Máximo & 24 & 24 & 24 & 16 & 16 & 16 & 16 & 16 & 16 \\
\hline \multicolumn{10}{|l|}{ Percentil } \\
\hline p. & 11 & 1 & 12 & 5 & 5 & 6 & 7 & 7 & 7 \\
\hline p15 & 13 & 14 & 14 & 6 & $r$ & 8 & 0 & 9 & 9 \\
\hline p25 & 14 & 15 & 14 & 7 & 8 & 8 & 9 & 9 & 9 \\
\hline p35 & 15 & 16 & 15 & 8 & 8 & 8 & 10 & 10 & 10 \\
\hline 150 & 16 & 17 & 16 & 8 & 9 & 9 & 10 & 11 & 11 \\
\hline p65 & 18 & 18 & 18 & 9 & 10 & 10 & 11 & 12 & 12 \\
\hline p75 & 18 & 18 & 18 & 10 & 10 & 10 & 12 & 12 & 12 \\
\hline por & 19 & 20 & 19 & 11 & 11 & 11 & 13 & 13 & 13 \\
\hline p95 & 22 & 22 & 21 & 12 & 12 & 13 & 15 & 15 & 15 \\
\hline
\end{tabular}

Nota: $M=$ Media; $D E=$ Desviación Estándar.

\section{Discusión}

El presente trabajo tuvo como objetivo adaptar y validar las escalas SIS/SES-SF en hombres y mujeres colombianos. Dicho objetivo se cumplió al realizar la validación de contenido y psicométrica de las escalas. Debido a la fiabilidad y validez que se han observado, puede decirse que tienen el sustento psicométrico adecuado para emplearse dentro de la población colombiana. Adicionalmente, su estructura trifactorial es equivalente al nivel estricto tanto en hombres como en mujeres. Las puntuaciones $V$ de Aiken dieron cuenta de un apropiado ajuste del contenido de las escalas al contexto colombiano. La evaluación cualitativa mostró que los ítems se ajustan de manera apropiada en términos de representatividad, comprensión, interpretación y claridad, lo que facilitaría la aplicación de la 
escala en la muestra y continuar con el proceso de validación.

En cuanto a redacción, la versión final colombiana de los ítems presenta algunas diferencias respecto a la española (cf., Moyano \& Sierra, 2014a). Se obtuvieron apropiados índices psicométricos de fiabilidad para la nueva versión colombiana de las escalas en cada factor, mostrando ser superiores a los obtenidos en la versión original (Carpenter et al., 2010), alemana (Rettenberger et al., 2019) y la española (Moyano \& Sierra, 2014a). Al igual que con la versión original (Carpenter et al., 2010) y las otras dos validaciones (i. e., española y alemana; Moyano \& Sierra, 2014a; Rettenberger et al., 2019), la conformación trifactorial fue observada en el AFC (i. e., el SES, SIS-1 y SIS-2). Como se esperaba, hubo correlación positiva entre los factores inhibitorios para ambos sexos. Las medias de los factores para hombres y mujeres fueron consistentes con los resultados mostrados por Carpenter et al. (2008), Moyano y Sierra (2014a) y Rettenberger et al. (2019), donde las mujeres suelen obtener puntuaciones inferiores a los hombres en el factor SES y superiores en los inhibitorios. Este patrón de resultados podría explicar la frecuencia de masturbación y al número de parejas sexuales observado en ambos sexos (Velten et al., 2018).

$\mathrm{El}$ análisis progresivo de la invarianza factorial mostró que la escala llegó a un nivel estricto de invarianza para ambos sexos, donde los indicadores de CFI y AIC no mostraron un decremento superior al 0.01 (IC $=90$ $\%)$, diferente a lo ocurrido en la versión alemana (Rettenberger et al., 2019). Esto facilita comparaciones por sexos con un mínimo sesgo en la medición (Dimitrov, 2010). El único peso factorial $(\lambda)$ inferior a 0.3 fue el del ítem 6 , similar a los resultados de la versión española (Moyano \& Sierra, 2014a), y cuya explicación podría deberse a que es el único referente a Infecciones de Trasmisión Sexual. Las SIS/SES-SF mostraron correlaciones con las otras escalas utilizadas. Las tres dimensiones del IDS se relacionaron con el factor SES como se esperaba (Bancroft \& Vukadinovic, 2004; Gomes et al., 2018; Janssen \& Bancroft, 2007; Santos-Iglesias \& Sierra, 2013;
Winters et al., 2008). Lo anterior puede aportar a la idea de la comprensión del deseo sexual como un componente cognitivo de la excitación sexual (Mitchell et al., 2014; Prause et al., 2007), sin conocer con claridad la dirección en la cual una pude influenciar sobre la otra. Diferente a lo observado por Moyano y Sierra (2014b), la propensión a la SI -en ambos factores- se relacionó significativa y negativamente con las dimensiones del IDS en ambos sexos; adicionalmente, la correlación entre el deseo y la SES podrían dar sustento a las dificultades autorreguladoras que algunos hombres tienen en el desempeño sexual (Nimbi et al., 2019) y que puede explicar las relaciones encontradas entre el PEDT y la SES. El funcionamiento sexual general (i. e., MGH-SFQ) correlacionó significativamente con la escala el SIS/SES-SF, lo cual sugiere la relevancia de las propensiones -inhibitorias o excitatorias- de la respuesta de excitación dentro de lo que tradicionalmente se enmarca en el ciclo de la respuesta sexual (Bancroft \& Janssen, 2000; Levin, 2017; Nolet et al., 2017).

La validación de las escalas es el inicio del estudio del MCD en Colombia y Latinoamérica dentro de las diferentes líneas de investigación en sexualidad humana (i. e., funcionamiento sexual, prevención de conductas sexuales de riesgo y abuso sexual); por lo que las correlaciones, aunque dan indicios interesantes para la comprensión de la excitación sexual colombiana, aún requiere de un trabajo más exhaustivo y minucioso para dar cuenta del fenómeno junto con un instrumento que lo evalúe de forma fiable y válida. Los resultados de esta investigación también dan cuenta del alcance generalizador y transcultural que implica el MCD en la población en general (Rettenberger et al., 2019; Sierra et al., 2018). De esta forma, para dar continuidad a esta línea, se recomienda realizar la posterior validación fisiológica del MCD que permita poner a prueba la capacidad predictiva de respuesta de las escalas en colombianos y, además, entender mejor los mecanismos neuropsicológicos que interactúan en la respuesta de inhibición y excitación sexual en diversas subpoblaciones colombianas. 
Por último, aunque las escalas parecen evaluar adecuadamente la propensión, no permite evaluar población clínica en Colombia, por lo que se recomienda hacer uso de instrumentos complementarios que permitan una evaluación y discriminación detallada de dificultades sexuales en contextos clínicos, además de poner a prueba este instrumento con población clínica en investigación.

\section{Referencias}

Aiken, L. R. (1985). Three coefficients for analyzing the reliability and validity of ratings. Educational Psychological Measurement, 45, 131-142. https://doi.org/1 $0.1177 / 0013164485451012$

American Educational Research Association, American Psychological Association, \& the National Council on Measurement in Education. (2014). Standards for educational and psychological tests. American Educational Research Association.

Bancroft, J., \& Janssen, E. (2000). The dual control model of male sexual response: A theoretical approach to centrally mediated erectile dysfunction. Neuroscience and Biobehavioral Reviews, 24, 571-579. https:// doi.org/10.1016/S0149-7634(00)00024-5

Bancroft, J., \& Vukadinovic, Z. (2004). Sexual addiction, sexual compulsivity, sexual impulsivity, or what? Toward a theoretical model. The Journal of Sex Research, 41, 225-234. https://doi.org/10.1080/00224490 409552230

Byrne, B. M., Oakland, T., Leong, F. T. L., van de Vijver, F. J. R., Hambleton, R. K., Cheung, F. M., \& Bartram, D. (2009). A critical analysis of cross-cultural research and testing practices: Implications for improved education and training in psychology. Training and Education in Professional Psychology, 3, 94-105. https://do i.org/10.1037/a0014516

Carpenter, D., Janssen, E., Graham, C. A., Vorst, H., \& Wicherts, J. (2008). Women's scores on the Sexual Inhibition/Sexual Excitation
Scales (SIS/SES): Gender similarities and differences. The Journal of Sex Research, 45, 36-48. https://doi.org/10.1080/0022449070 1808076

Carpenter, D. L., Janssen, E., Graham, C. A., Vorst, H., \& Wicherts, J. (2010). The Sexual Inhibition/Sexual Excitation ScalesShort Form (SIS/SES-SF). En T. D. Fisher, C. M. Davis, W. L. Yarber \& S. L. Davis (Eds.), Handbook of sexuality-related measures (pp. 236-239). Routledge.

Carvalho, J., Quinta-Gomes, A., \& Nobre, P. J. (2013). The sex functioning profile of a nonforensic simple of individuals reporting sexual agression against women. Journal of Sex Medicine, 10, 1744-1754. https://doi.or $\mathrm{g} / 10.1111 / \mathrm{jsm} .12188$

Cheung, G. W., \& Rensvold, R. B. (2002). Evaluating goodness-of-fit indexes for testing measurement invariance. Structural Equation Modeling, 9, 233-255. https://doi.o rg/10.1207/S15328007SEM0902_5

Dèttore, D., Pucciarelli, M., \& Santarnecchi, E. (2013). Anxiety and female functioning: An empirical study. Sex and Marital Therapy, 39, 216-240. https://doi.org/10.1080/00922 623X.2011.606879

Dimitrov, D. M. (2010). Testing for factorial invariance in the context of construct validation. Measurement and Evaluation in Counseling and Development, 43, 121-149. $\mathrm{h}$ ttps://doi.org/10.1177/0748175610373459

Elosua, P., Mujika, J., Almeida, L. S., \& Hermosilla, D. (2014). Procedimientos analítico-racionales en la adaptación de test. Adaptación al español de la batería de pruebas de razonamiento. Revista Latinoamericana de Psicología, 46, 117-126. https://doi.org/10.1016/S0120-05 34(14)70015-9

Fava, M., Rankin, M. A, Alpert, J. E, Nierenberg, A. A., \& Worthington, J. J. (1998). An open trial of oral sildenafil in antidepressantinduced sexual dysfunction. Psychotherapy and Psychosomatics, 67, 328-331. https://doi .org/10.1159/000012299

Gomes, A. L. Q., Janssen, E., Santos-Iglesias, P., Pinto-Gouveia, J., Fonseca, L. M., \& 
Nobre, P. J. (2018). Validation of the Sexual Inhibition and Sexual Excitation Scales (SIS/SES) in Portugal: Assessing gender differences and predictors of sexual functioning. Archives of Sexual Behavior, 47, 1721-1732. https://doi.org/10.1007\%2Fs10 508-017-1137-8

Gray, J. A. (1987). The psychology of fear and stress. Cambridge University Press

Janssen, E., \& Bancroft, J. (2007). The dual control model: The role of sexual inhibition and excitation in sexual arousal and behavior. In E. Janssen (Ed.), The Kinsey Institute series. The psychophysiology of sex (p. 197-222). Indiana University Press.

Janssen, E., Vorst, H., Finn, P., \& Bancroft, J. (2002a). The Sexual Inhibition (SIS) and Sexual Excitation (SES) Scales: I. Measuring sexual inhibition and excitation proneness in men. The Journal of Sex Research, 39, 114-126. https://doi.org/10.10 80/00224490209552130

Janssen, E., Vorst, H., Finn, P., \& Bancroft, J. (2002b). The Sexual Inhibition (SIS) and Sexual Excitation (SES) Scales: II. Predicting psychophysiological response patterns. The Journal of Sex Research, 39, 127-132. https://doi.org/10.1080/00224490 209552131

Kinsey, A. C., Pomeroy, W. B., \& Martin, C. E. (1948). Sexual behavior in the human male. W. B. Saunders Co.

Kilimnik, C. D., \& Meston, C. M. (2016). Role of body esteem in the sexual excitation and inhibition responses of women with and without a history of childhood sexual abuse. The Journal of Sexual Medicine, 13, 1718-1728. https://doi.org/10.1016/j.jsxm. 2016.09.004

Levin, R. J. (2017). The human sexual response cycle. En W.W. IsHak (Ed.), The textbook of clinical sexual medicine (pp. 9-51). Springer International Publishing.

Lorenzo-Seva, U., \& Ferrando, P. J. (2015). POLYMAT-C: A comprehensive SPSS program for computing the polychoric correlation matrix. Behavior Research
Methods, 47, 884-889. https://doi.org/10.37 58/s13428-014-0511-x

Malavige, L. S., Wijesekara, P. N., Jayaratne, S. D., Kathriarachchi, S. T., Ranasinghe, P., Sivayogan, S., Levi, J. C., \& Bancroft, J. (2013). Linguistic validation of the Sexual Inhibition and Sexual Excitation Scales (SIS/SES) translated into five South Asian languages: Oxford Sexual Dysfunction Study (OSDS). BMC Research Notes, 6, 550. https://doi.org/10.1186/1756-0500-6550

Marchal-Bertrand, L., Espada, J. P., Morales, A., Gómez-Lugo, M., Soler, F., \& Vallejo-Medina, P. (2016). Adaptación, validación, fiabilidad del Massachussets General Hospital-Sexual Functioning Questionnaire en una muestra colombiana y equivalencia factorial con la versión española. Revista Latinoamericana de Psicología, 48(2), 88-97. https://doi.org/10.1 016/j.rlp.2016.01.001

Merino, C., \& Livia, J. (2009). Intervalos de confianza asimétricos para el índice la validez de contenido: un programa Visual Basic para la V de Aiken. Anales de Psicología, 25, 169-171. http://revistas.um.e s/analesps

Mitchell, K. R.., Wellings, K. A., \& Graham, C. (2014). How do men and women define sexual desire and sexual arousal? Journal of Sex Eु Marital Therapy, 40, 17-32. https://d oi.org/10.1080/0092623X.2012.697536

Montero, I., \& León, O. G. (2007). A guide for naming research studies in Psychology. International Journal of Clinical and Health Psychology, 7, 847-862. http://www.aepc.es/ ijchp/GNEIP07_es.pdf

Moyano, N., \& Sierra, J. C. (2014a). Validación de las Escalas de Inhibición Sexual/Excitación Sexual-Forma Breve (SIS/SES-SF). Terapia Psicológica, 32, 87-100. http://www.scielo.cl/scielo.php?pid $=\mathrm{S} 0718-48082014000200002 \& \mathrm{script}=\mathrm{sci}$ arttext

Moyano, N., \& Sierra, J. C. (2014b). Funcionamiento sexual en hombres y mujeres víctimas de abuso sexual en 
la infancia y en la adolescencia/adultez. Revista Internacional de Andrología, 12, 132-138. https://doi.org/10.1016/j.androl.2 014.04 .012

Moyano, N., Vallejo-Medina, P., \& Sierra, J. C. (2016). Sexual Desire Inventory: Two or three dimensions? The Journal of Sex Research, 54(1), 105-116. https://doi.org/10 $.1080 / 00224499.2015 .1109581$

Muñiz, J., Elosua. P., \& Hambleton, R. K. (2013). Directrices para la traducción y adaptación de los tests: segunda edición. Psicothema, 25, 151-157. https://doi.org/10.7334/psicot hema2013.24

Nimbi, F. M., Tripodi, F., Rossi, R., Michetti, P. M., \& Simonelli, C. (2019). Which psychosocial variables affect drive the most? Analysis of sexual desire in a group of Italian men. International Journal of Impotence Research, 31, 410-423. https://doi.org/10.10 38/s41443-018-0105-8

Nolet, K., Wilson, A. L., \& Rouleau, J. L. (2017). Using the dual control model to understand problematic sexual behaviors in men. Sexologies, 26, e55-e58. https://doi.org /10.1016/j.sexol.2017.09.001

Osterlind, S. J. (1989). Constructing test items: Multiple-choice, constructed-response, performance, and other formats. Kluwer Academic Publishers. https://doi.org/10.10 07/978-94-009-1071-3_1

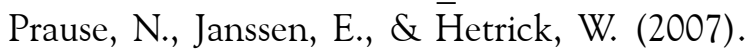
Attention and emotional responses to sexual stimuli and their relationship to sexual desire. Archives of Sexual Behavior, 37, 934-949. https://doi.org/10.1007/s1050 8-007-9236-6

Pulverman, C. S., \& Meston, C. M. (2020). Sexual dysfunction in women with a history of childhood sexual abuse: The role of sexual shame. Psychological Trauma: Theory, Research, Practice, and Policy, 12, 291-299. h ttps://doi.org/10.1037/tra0000506

Rettenberger, M., de Albuquerque Camarão, B., Breiling, L., Etzler, S., Turner, D., Klein, V., \& Briken, P. (2019). A validation study of the German version of the Sexual Inhibition/Sexual Excitation Scales-Short
Form. Archives of Sexual Behavior, 48, 2553-2563. https://doi.org/10.1007/s10508 -019-01489-w

Rettenberger, M., Klein, V., \& Briken, P. (2015). The relationship between hypersexual behavior, sexual excitation, sexual inhibition, and personality traits. Archives of Sexual Behavior, 45, 219-233. ht tps://doi.org/10.1007/s10508-014-0399-7

Rosen, R., Cappelleri, J., Smith, M., Lipsky, J., \& Peña, B. (1999). Development and evaluation of an abridged, 5item version of the International Index of Erectile Function (IIEF-5) as a diagnostic tool for erectile dysfunction. International Journal of Impotence Research, 11, 319-326. http://patientreportedoutcom es.ca/files/2014/04/IIEF-5-Rosen-1999.pdf

Sanders, S. A., Graham, C. A., \& Milhausen, R. R. (2008). Predicting sexual problems in women: The relevance of sexual excitation and sexual inhibition. Archives of Sexual Behavior, 37, 241-251. https://doi.org/10.10 07/s10508-007-9235-7

Santos-Iglesias, P., \& Sierra, J. C. (2013). Predictors of sexual assertiveness: The role of sexual desire, arousal, attitudes, and partner abuse. Archives of Sexual Behavior, 42, 1043-1052. https://doi.org/10.1007/s10 508-012-9998-3

Sarin, S., Amsel, R., \& Binik, Y. M. (2015). A streetcar named "derousal"? A psychophysiological examination of the desire-arousal distinction in sexually functional and dysfunctional women. The Journal of Sex Research, 53, 711-729. https:/ /doi.org/10.1080/00224499.2015.1052360

Sierra, J. C., De la Rosa Centella, M. D., Granados, R., Calvillo, C., Arcos-Romero, A. I., Sánchez-Fuentes, M. D. M., \& Moyano, N. (2018). Evidencias de validez de la versión española de las Sexual Inhibition/Sexual Excitation Scales-Short Form (SIS/SES-SF). Revista Iberoamericana de Diagnóstico y Evaluación, 50, 173-184. ht tps://doi.org/10.21865/RIDEP50.1.14

Spector, I. P., Carey, M. P., \& Steinberg, L. (1996). The sexual desire inventory: Development, 
factor structure, and evidence of reliability. Journal of Sex 8 Marital Therapy, 22, 175-190. https://doi.org/10.1080/00926239 608414655

Symonds, T., Perelman, M. A., Althof, S., Giuliano, F., Martin, M., May, K., \& Morris, M. (2007). Development and validation of a Premature Ejaculation Diagnostic Tool. European Urology, 52, 565-573. http://www.shimclinic.com/docu ments/PIIS0302283807000322.pdf

Tavares, I. M., Laan, E. T., \& Nobre, P. J. (2018). Sexual inhibition is a vulnerability factor for orgasm problems in women. The Journal of Sexual Medicine, 15, 361-372. https://doi.or g/10.1016/j.jsxm.2017.12.015

Turner, D., Briken, P., Klein, V., \& Rettenberger, M. (2013). SIS/SESSF-Sexual Inhibition/ Sexual Excitation Scales-Short Form. En B. Strauß, E. Brähler \& D. Richter (Eds.), Sexualwissenschaftliche Testverfahren [Sexological Questionnaires] (pp. 168-172). Hogrefe.

Vallejo-Medina, P., Gómez-Lugo, M., MarchalBertrand, L., Saavedra-Roa, A., Soler, F., \& Morales, A. (2017). Developing guidelines for adapting questionnaires into the same language in another culture. Terapia Psicológica, 35, 181-194. http://www .redalyc.org/html/785/78552281004/

Vallejo-Medina, P., Saavedra-Roa, A., \& PérezDurán, C. (2017). Translation, adaptation, and preliminary validation of the Female Sexual Function Index into Spanish (Colombia). Archives of Sex Research, 47, 797-810. https://doi.org/10.1007/s10508-0 17-0976-7

Vallejo-Medina, P., Peñuela-Trujillo, I., Saffon, J. P., \& Álvarez-Muelas, A. (2020). Spanish validation of the International Index of Erectile Function (IIFE-5). (en revisión).

Vallejo-Medina, P., Rojas-Paoli, I., \& ÁlvarezMuelas, A. (2020). Validation of the Sexual Desire Inventory in Colombia. Journal of Sex Eु Marital Therapy, 46, 385-398. https://doi .org/10.1080/0092623X.2020.1739181

Vallejo-Medina, P., Saffon, J. P., PeñuelaTrujillo, C. \& Álvarez-Muelas, A. (2020c).
Adaptation, and clinical validation of Premature Ejaculation Diagnostic Tool (PEDT) in Spanish. (en revision).

van Lankveld, J. J. D. M., Platteau, T., van Montfort, K., Nieuwenhuijs, F., \& Syroit, J. (2014). The predictive validity of SIS/ SES and BIS/BAS scores for sexual and non-sexual risk behavior. Personality and Individual Differences, 79, 7-12. https://doi.o rg/10.1016/j.paid.2015.01.048

Varjonen, M., Santtila, P., Höglund, M., Jern, P., Johansson A., Wager, I., Witting, K., Ålgars, M., \& Sandnabba, M. K. (2007). Genetic and environmental effects on sexual excitation and sexual inhibition in men. The Journal of Sex Research, 44, 359-369. https://doi.org/10.1080/00224490 701578653

Velten, J. (2017). The dual control model of sexual response: Relevance of sexual excitation and sexual inhibition for sexual function. Current Sexual Health Reports, 9, 90-97. https://doi.org/10.1007/s11930-0170108-3

Velten, J., Scholten, S., \& Margraf, J. (2018). Psychometric properties of the Sexual Excitation/Sexual Inhibition Inventory for Women and Men (SESII-W/M) and the Sexual Excitation Scales/Sexual Inhibition Scales short form (SIS/SES-SF) in a population-based sample in Germany. PloSOne, 13. https://doi.org/10.1371/journ al.pone. 0193080

Winters, J., Christoff, K., \& Gorzalka, B. B. (2008). Conscious regulation of sexual arousal in men. Journal of Sex Research, 46, 330-343. https://doi.org/10.1080/00224490 902754103

\section{Apéndice}

Sexual Inhibition/Sexual Excitation Scales-Short Form ([SIS/SES-SF]; Carpenter, Janssen, Graham, Vorst, \& Wicherts, 2010) - Versión validada en Colombia 
2 Con la autorización http://www.pfizerpatien treportedoutcomes.com
En este cuestionario se encuentran afirmaciones sobre cómo podría ser su reacción ante diversas situaciones, actividades sexuales o comportamientos. Obviamente, cuál sea su reacción, a menudo, depende de las circunstancias. Por favor, lea cada afirmación cuidadosamente y elija cuál sería su reacción más probable. Seleccione la opción que corresponda. En ocasiones, puede sentir que ninguna de las respuestas parece completamente precisa o puede leer una afirmación en la que sienta que esto "no aplica" para usted. Si es así, por favor marque la respuesta que usted elegiría en el caso de que sí aplicara. No piense demasiado para dar su respuesta. Marque siempre la opción que considere más válida para usted. Trate de no saltarse ninguna pregunta. Trate de ser lo más honesto posible.

\section{Notas}

* Artículo de investigación. Este trabajo ha sido realizado gracias a la financiación otorgada por la Fundación Universitaria Konrad Lorenz asociada al proyecto de investigación número: 2015-009 55270152 concedido a Pablo Vallejo Medina.

1 Con la autorización http://www.pfizerpatien treportedoutcomes.com 